\title{
The magnetic circuit dynamics of a magnetorheological valve with a permanent magnet
}

\author{
Michal Kubík ${ }^{1, *}$, Filip Jeniš ${ }^{1}$, and Igor Hašlík ${ }^{1}$ \\ ${ }^{1}$ Brno University of Technology, Institute of Machine and Industrial Design, Technická 2, Brno, \\ Czech Republic
}

\begin{abstract}
The magnetorheological (MR) damper uses magnetorheological fluid which, when subjected to magnetic stimuli, generates an increase of damping forces. A significant problem of these dampers is their poor failsafe ability due to power supply interruption. In the case of faults, the damper remains in a low damping state, which is dangerous. This problem can be solved by accommodating a permanent magnet in the magnetic circuit of the damper. However, the magnetic circuit dynamic of this type of damper has rarely been studied. The main aim of this paper is to introduce the magnetic circuit dynamics of the magnetorheological damper/control valve with a permanent magnet. Firstly, the design of the magnetorheological valve with $\mathrm{NdFe} 42$ permanent magnet in the magnetic circuit is introduced. The response time of the magnetic field on the unit step of the control signal was calculated by transient magnetic simulation in Ansys Electronics software. The response time of the magnetic field was simulated in the range of 1.2 to $5 \mathrm{~ms}$ depending on the electric current magnitude and orientation. The presented MR damper was manufactured and tested. The experiments prove that the permanent magnet significantly affects the dynamics of the magnetic circuit.
\end{abstract}

\section{Introduction}

The magnetorheological (MR) damper utilizes magnetorheological (MR) fluid which, when subjected to magnetic stimuli, generates yield stress thus increasing its apparent viscosity [1]. MR fluid is a suspension of microsized ferromagnetic particles which are dispersed in the carrier fluid. The change of apparent viscosity is caused by particles aligning in the direction of the magnetic field. Several designs of the MR damper can be found that differ in the number of coils $[2,3,4]$, the arrangement of the magnetic circuit [3, $4,5]$ and the number of gaps [6,7] etc. If the power supply of the electromagnetic coil is interrupted during damper operation, the damper will remain at the minimum damping level. This is a significant problem for a wide range of MR damper applications (aerospace, rail, etc.). A permanent magnet accommodated into the MR damper magnetic circuit is a

\footnotetext{
*Corresponding author: michal.kubik@ vutbr.cz
} 
possible solution. The permanent magnet creates a magnetic flux in the magnetic circuit, which ensures a sufficient damping level during a power supply failure. In some published designs, the permanent magnet is placed under the inner edge of the electromagnetic coil $[8,9,10,11]$ or next to the electromagnetic coil on the edge of the piston gap $[12,13]$. The damping level with no electric current and with a permanent magnet usually achieved onethird of the maximum damping force at a given piston velocity. A low-coercivity permanent magnet (e.g. Alnico) accommodated in the magnetic circuit of a MR damper is an interesting idea for this purpose [12]. However, using a so-called switchable magnet does not ensure the full fail-safe ability of the MR damper due to the possibility of magnet demagnetisation.

Only a few authors have dealt with the dynamic behavior of MR dampers. Koo et al. [14] experimentally determined the response time of a commercially available Lord MR damper at approximately $17 \mathrm{~ms}$ (lowest measured value). However, the response time of the damper is strongly dependent on the applied electric current level and the piston velocity. Goldasz et al. [15] experimentally determined the response time of three commercially available MR dampers using a sensorless measurement method based on voltage and current measurements. Takesue et al. [16] presented the design of the MR clutch where they improved the dynamic behavior of this actuator through a reduction of the eddy current in the magnetic circuit. Strecker et al. [17] and Kubík et al. [4] published the design of the MR damper with a short response time in which the ferrite material of the magnetic circuit was used. Strecker et al. [5] published an MR damper with a magnetic circuit manufactured by 3D metal printing. This design method provides a MR damper with a short response time of $1.68 \mathrm{~ms}$ and a high dynamic force range. This method is based on the reduction of the eddy current in the magnetic circuit. The dynamic behavior of the MR damper with a permanent magnet has only beed studied by Lee et al. [18]. The damping force of this damper with a magnet is realised by the variation of the magnetisation area (position-dependent damper), not by the electromagnetic coil.

The main aim of this paper is to introduce the magnetic circuit dynamics (magnetic field response time) of the MR damper (control valve) with a permanent magnet.

\section{Materials and methods}

\subsection{The design of a magnetorheological damper with a permanent magnet}

The single-tube configuration of the MR damper in flow-mode is presented, see Fig. 1. The floating piston (3) separates the Lord MRF 132-DG MR fluid (5) from the high-pressure nitrogen in the reservoir (2). The piston rod seal and bearing are accommodated in part (4). The MR control valve (1) creates a damping effect. The control valve consists of a core (8, 9), sleeve (10), piston rod (12), lids (11), permanent magnet (6), and an electromagnetic coil (7), see Fig. 1. The magnetic circuit $(8,9,10)$ was made from $11 \mathrm{SMn} 30$ cutting steel. Two rings of neodymium permanent magnets (NdFe42) measuring 25x16x5 mm were used. An electromagnetic coil was wound with 190 turns of copper wire with a diameter of $0.5 \mathrm{~mm}$. In the case of the positive polarity of the electric current $+I$, the magnetic flux density in the gap is $B_{\max }=B_{\max }+B_{\text {coil }}$. In the case of the negative polarity of electric current $-I$, the magnetic flux density in the gap is $B_{\min }=B_{\max }-B_{\text {coil }}$, see Fig. 1 . 


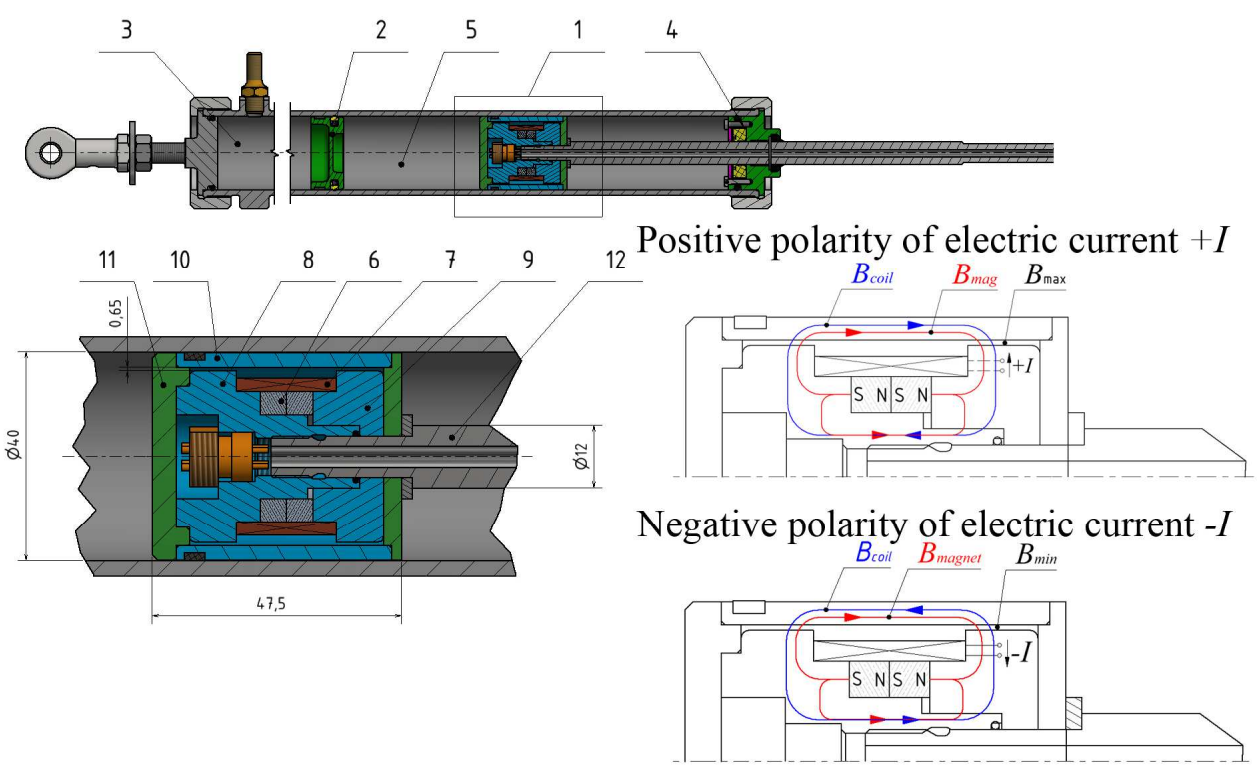

Fig. 1. Design of MR damper/control valve with the permanent magnet.

\subsection{Magnetic model}

The Ansys Electronics Desktop 2018 with co-simulation of Ansys Twin Builder 2018 was used for the presented magnetic model. We assumed that the magnetorheological control valve in the damper was axially symmetrical around the centre line $\mathrm{Z}$ in a cylindrical coordinate system. The simplified geometry used in the magnetic simulation can be seen below.
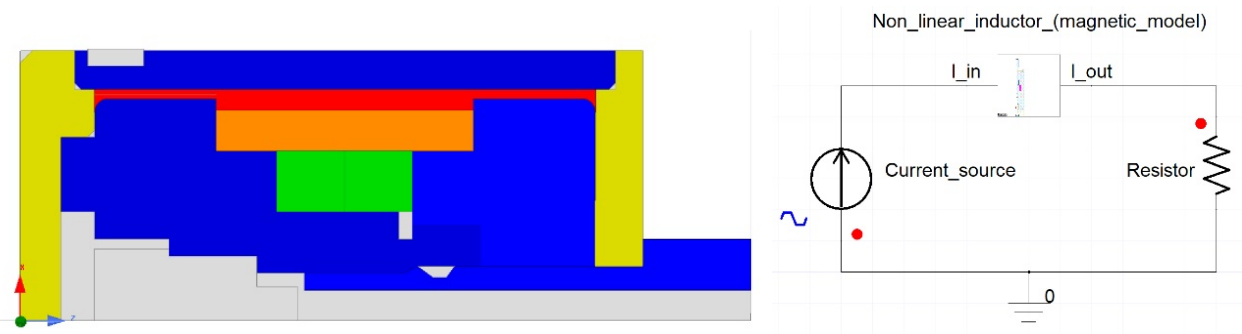

Fig. 2. Simplified geometry of MR control valve (left) - 11SMn30 - blue, MRF/air - red, copper orange, magnet - green, bronze - yellow; lumped electric circuit (right).

The transient magnetic simulation was connected to the external electric circuit, see Fig. 2. The lumped electric circuit consists of an ideal electric current source (control input), resistor (coil winding), and MR control valve object (non-linear inductor). The virgin and hysteresis magnetisation curves of used materials are very important inputs for the model. Therefore, experimental determination was necessary. The magnetisation curves were measured by system Remagraph C-500. The coercivity and remanence were determined from the measured hysteresis loop of $11 \mathrm{SMn} 30$ material: $\mathrm{H}_{\mathrm{c}}=427 \mathrm{~A} / \mathrm{m}$ and $\mathrm{B}_{\mathrm{r}}=1.05 \mathrm{~T}$. The electric bulk conductivity of $5.8 \mathrm{MS} / \mathrm{m}$ was used in accordance with the datasheet from the steel supplier. It was used the vector hysteresis modeling feature available in system 
Ansys for a magnetic model of the MR control valve. A more detailed description of this method is available in the document [1]. The presented transient magnetic model calculates the magnetic flux density response in the gap (air / MRF) on the current unit-step. The final value of the electric current was reached at $0.2 \mathrm{~ms}$ in the previously performed experiments. Therefore, this value is assumed as the rise time of the control signal in the magnetic simulation. The constant simulation time step was set at $0.2 \mathrm{~ms}$. The mesh was generated with refinement on the edges of the magnetic circuit due to the eddy currents effect.

\subsection{Measurement of magnetic circuit dynamics with air in the gap}

The measurement was performed for verification of transient magnetic models with air in the gap. The correct value of magnetic flux density in the gap can be measured only in the case of relative permeability $\mu_{\mathrm{r}}=1$ (air) in the gap using a Hall probe because its permeability is similar to $\mu_{\mathrm{r}}$. It can be assumed that the magnetic model verified with air in the gap is also valid for MR fluid in the gap. The course of magnetic flux density on the current unit-step was measured. The primary $\tau_{63}, \tau_{36}(63.2 \%$ or $36.8 \%$ of the steady-state value) and secondary $\tau_{90}, \tau_{10}(90 \%$ or $10 \%$ of the steady-state value) response times of the magnetic field were determined from the measured data. Our patented current controller working with the overvoltage method was used for the response time measurement [17]. The controller was powered by a laboratory supply and the input signal was generated from the Arduino control board. The experimental setup diagram can be seen in Fig. 3. The magnetic flux density in the gap was measured with a magnetometer (F.W. Bell 5180) with an ultra-thin transverse probe (STB1X-0201). The course of the electric current was measured with a Fluke i30s current clamp. These two signals were measured with a sampling frequency of $200 \mathrm{kHz}$ using a Dewetron USB-50 front-end analyser. The measured data was processed using DeweSoft software. The course of magnetic flux density in the gap was measured for two levels of electric current ( $1 \mathrm{~A}$ and $2 \mathrm{~A}$ ) and also for opposite polarity ( $-1 \mathrm{~A}$ and $-2 \mathrm{~A})$. The rise and drop of magnetic flux density was measured for each magnitude of electric current.
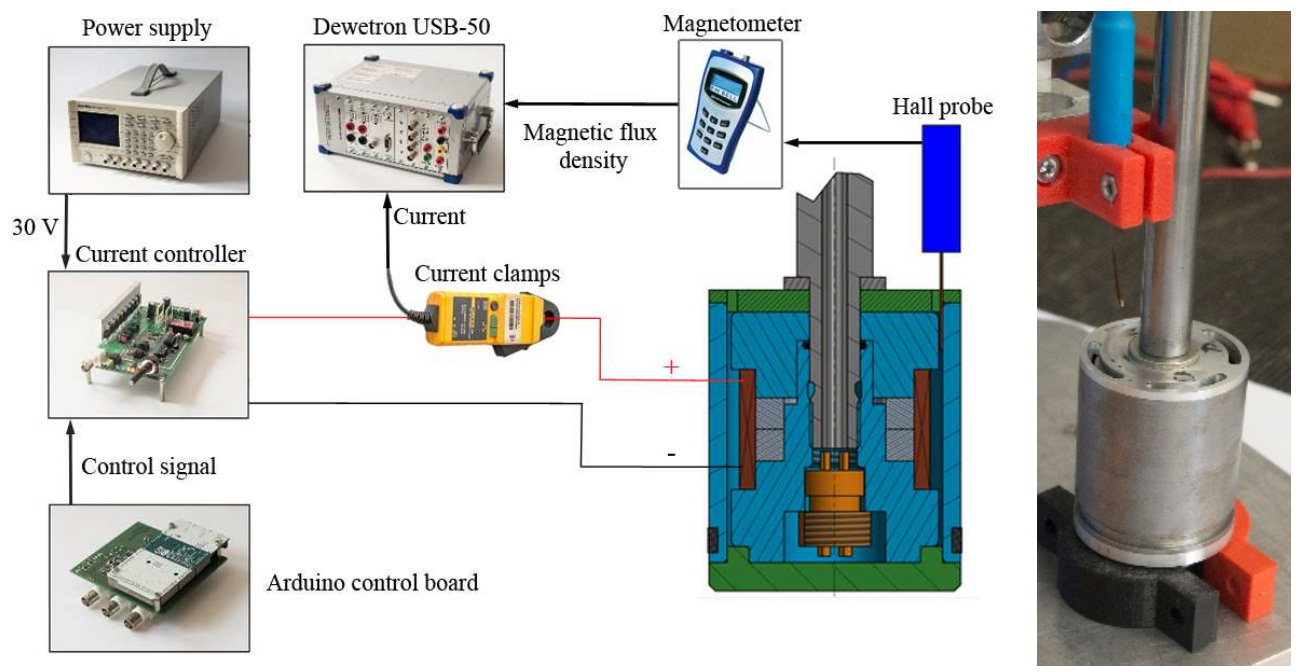

Fig. 3. Experimental setup diagram. 


\section{Results and discussion}

\subsection{Magnetic circuit dynamics with air in the gap}

The response of magnetic flux density $B$ on the unit-step of the control signal from $I=O A$ to $I=-1$ A determined from the magnetic model (red) and experiment (blue) can be seen in Fig. 4. The steady-state magnetic flux density determined from the magnetic model differs from the measured data. The difference for the electric current $-1 \mathrm{~A}$ is $12.5 \%$ (see time 0 $s$ at Fig. 4). This error may be explained by inaccuracies in the magnetisation curve of the permanent magnet and the cutting steel. All collected data of magnetic flux density $B$ (induction) courses from the model and the experiment were normalised, see Fig. 4.
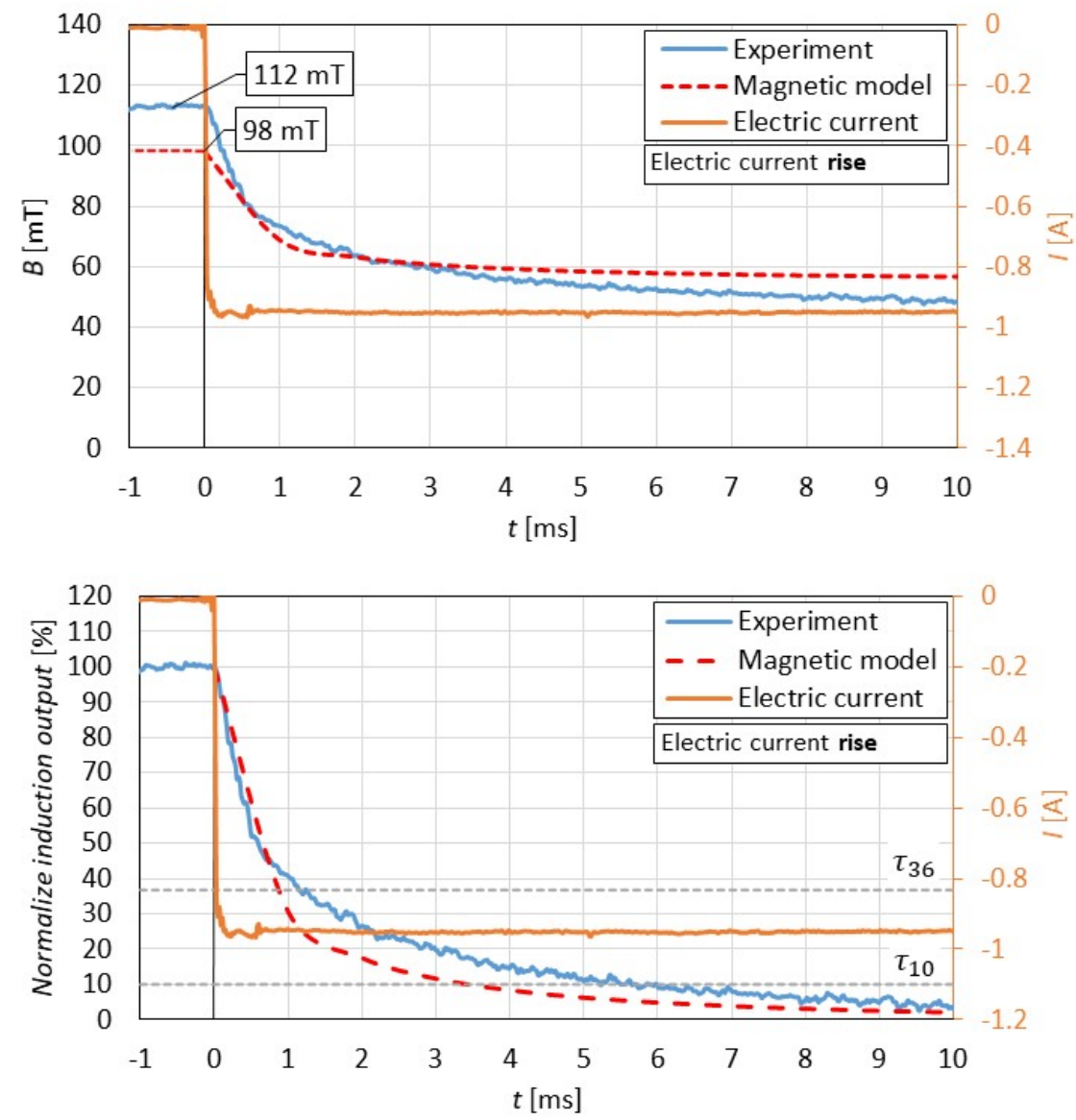

Fig. 4. The course of magnetic flux density $B$ for the unit step of electric current -1 A from the magnetic model (red) and experiment (blue) - an example of the determination method of response time.

The primary $\tau_{63}, \tau_{36}$, and secondary $\tau_{90}, \tau_{10}$ response times were determined from simulation and experimental data, see Fig. 5. The response time of magnetic flux density $B$ for the opposite polarity $(-I)$ of electric current is significantly lower than for positive polarity $(+I)$. The primary response time for the electric current rise from $I=0 A$ to $I=-2 A$ is $\tau_{63}=0.95$ $\mathrm{ms}$ and the electric current drop from $I=-2 A$ to $I=O A$ is $\tau_{36}=0.97 \mathrm{~ms}$. The primary 
response time for the electric current rise from $I=O A$ to $I=2 \mathrm{~A}$ is $\tau_{63}=4.6 \mathrm{~ms}$ and the drop from $I=2 \mathrm{~A}$ to $I=O \mathrm{~A}$ is $\tau_{36}=3.6 \mathrm{~ms}$. The magnetic model describes the primary response time very well. The secondary response time exhibits greater variance especially in the case of an electric current drop, see Fig. 5.
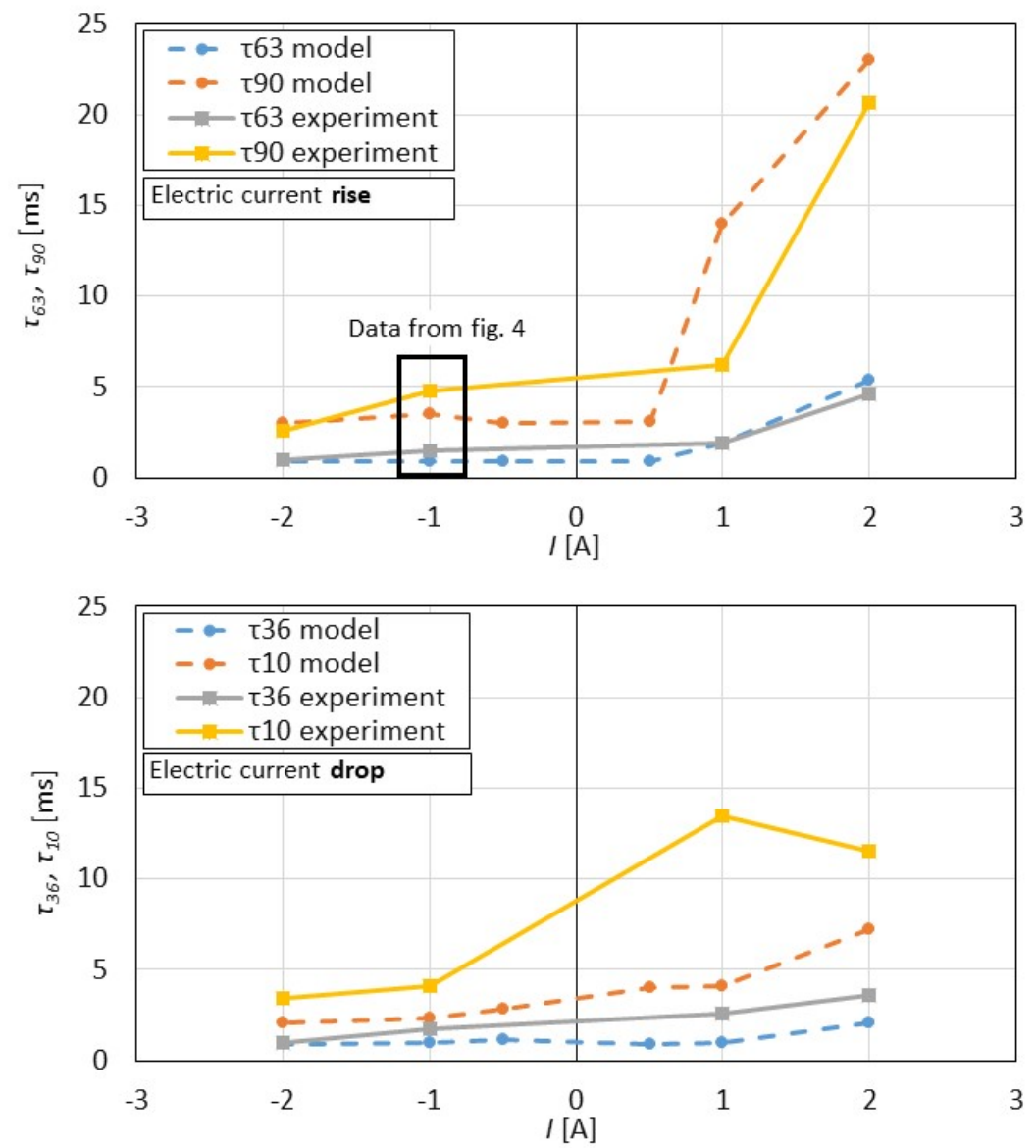

Fig. 5. The primary $\tau_{63}, \tau_{36}$ and secondary $\tau_{90}, \tau_{10}$ response time dependency on the magnitude and orientation of electric current for the rise and drop in electric current for air in the gap (square = experiment, circle $=$ model $)$.

\subsection{Magnetic circuit dynamics with MRF in the gap}

The experimentally verified magnetic model was used to determine the dynamic behaviour (the response time of the magnetic field) of the magnetic circuit with MR fluid in the gap. The magnetic field response time is the largest proportion of the total MR damper response time. Thus, it can be assumed that the response time determined from the transient magnetic model (Fig. 6) is near to the force response time of the MR damper. The orientation and magnitude of the electric current significantly affect the course of primary and secondary response time on electric current, see Fig. 6. A significantly lower response time occurs for the opposite polarity $-I$ of electric current and reaches the value of $\tau_{63}=1.1$ $\mathrm{ms}$ for an electric current rise from $I=O A$ to $I=-l$ A. This value is almost independent of the electric current magnitude. The primary and secondary response time is strongly 
dependent on the electric current magnitude in the case of positive polarity $+I$ of electric current, see Fig. 6 . The primary response time reaches $\tau_{63}=17 \mathrm{~ms}$ for an electric current rise from $I=O A$ to $I=1 \mathrm{~A}$ and $\tau_{36}=14.5 \mathrm{~ms}$ for an electric current rise from $I=1 \mathrm{~A}$ to $I=0 \mathrm{~A}$. The presented MR damper is over 13 times faster for the negative polarity of electric current than for positive. The permanent magnet significantly influences the dynamic behaviour of the magnetic circuit. This means that the MR dampers have a short response time for the transient from a fail-safe state to low damping forces and a long response time for the transient to high damping forces.
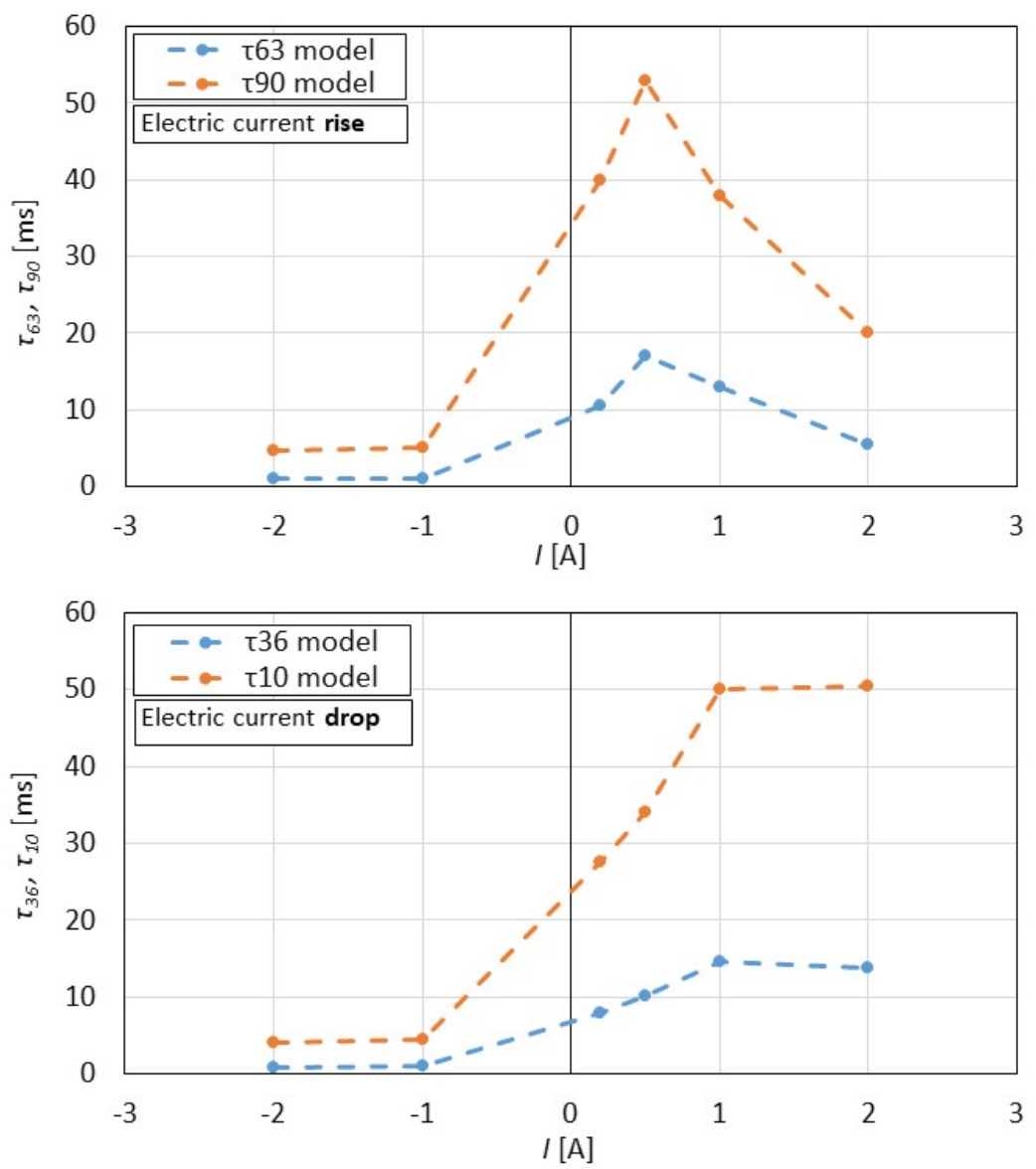

Fig. 6. The primary $\tau_{63}, \tau_{36}$ and secondary $\tau_{90}, \tau_{10}$ response time dependency on the magnitude and orientation of electric current for the rise and drop in electric current for $M R F$ in the gap.

\section{Conclusion}

This paper deals with the dynamic behaviour of the magnetic circuit of the MR control valve with a permanent magnet. The response times of the MR damper were determined from the magnetic model and the experiment. The conclusions from this paper are as follows: 
- The primary and secondary response time of the magnetic field on the unit-step of the control signal is significantly faster for the negative polarity of electric current -I (-2 A; $\tau 63=0.95 \mathrm{~ms}, \tau 36=0.97 \mathrm{~ms})$ than for positive $+\mathrm{I}(2 \mathrm{~A} ; \tau 63=4.6 \mathrm{~ms}, \tau 36=3.6 \mathrm{~ms})$ with an air gap, see Fig. 5. The trends are similar in the case of MRF in the gap (Fig. 6).

- The dynamic behaviour of the magnetic field determined from the magnetic model fitted well the experimental data (Fig. 5). For this reason, a transient magnetic model is an effective tool for the determination of the dynamic behaviour of the magnetic circuit of the MR control valve. However, the secondary response time exhibits greater variance.

- The permanent magnet significantly influences the dynamic behaviour of the magnetic circuit of the MR control valve/damper.

Further research will be focussed on the determination of the MR damper force-velocity map and the measurement of force response time.

The authors wish to acknowledge the support of grant no. PPI//APM/2018/1/00027/DEC/1, National Agency for Academic Exchange, Poland, and FEKT/FSI-J-20-6260, GAČR 20-23261Y and FSI-S20-6247.

\section{References}

1. M. Kubík, J. Goldasz, Shock Vib. 2019, 20 (2019)

2. J. Zheng, Y. Li, J. Wang, Proc. Inst. Mech. Eng. Part C J. Mech. Eng. Sci. 231, 16 (2017)

3. J. Gołdasz, Prz. Elektrotechniczny 89, (2013) (in Polish)

4. M. Kubík, O. Macháček, Z. Strecker, J. Roupec, I. Mazůrek, Smart Mater. Struct. 26, 9 (2017)

5. Z. Strecker, M. Kubík, P. Vitek, J. Roupec, D. Paloušek, V. Šreibr, Smart Mater. Struct. 28, 13 (2019)

6. J. Goldasz, Proceedings of the 20th International Conference on Research and Education in Mechatronics (2019)

7. J. Goldasz, Int. J. Veh. Des. 62, 20 (2013)

8. X. X. Bai, N. M. Wereley, J. Appl. Phys. 115, 4 (2014)

9. H. H. Zhang, C. R. Liao, M. Yu, S. L. Huang, Smart Mater. Struct. 16, 8 (2007)

10. W. Yan, J. Ji, B. Dong, H. Ge, Struct. Control Heal. Monit. 18, 19 (2009)

11. C. Du, F. Wan, G. Yu, Smart Mater. Struct. 20, 10 (2011)

12. H. Böse, J. Ehrlich, J. Intell. Mater. Syst. Struct. 23, 8 (2012)

13. H. Bose, J. Ehrlich, A.-M. Trendler, 11th Conference on Electrorheological Fluids and Magnetorheological Suspensions (2009)

14. J. H. Koo, F. D. Goncalves, M. Ahmadian, Smart Mater. Struct. 15, 7 (2006)

15. J. Gołdasz, B. Sapinski, Ł. Jastrze, Shock Vib. 2018, 21 (2018)

16. N. Takesue, Y. Kiyota, J. Furusho, Proceedings of the 41st SICE Annual Conference, (2020)

17. Z. Strecker, J. Roupec, I. Mazurek, O. Machacek, M. Kubik, M. Klapka, J. Intell. Mater. Syst. Struct. 26, 7 (2015)

18. T. H. Lee, S. B. Choi, Smart Mater. Struct. 28, 16 (2019) 